\title{
A Random Holding Period Approach for Liquidity-Inclusive Risk Management
}

\author{
Damiano Brigo and Claudio Nordio
}

\begin{abstract}
Within the context of risk integration, we introduce risk measurement stochastic holding period (SHP) models. This is done in order to obtain a 'liquidityadjusted risk measure' characterized by the absence of a fixed time horizon. The underlying assumption is that-due to changes in market liquidity conditions-one operates along an 'operational time' to which the $\mathrm{P} \& \mathrm{~L}$ process of liquidating a market portfolio is referred. This framework leads to a mixture of distributions for the portfolio returns, potentially allowing for skewness, heavy tails, and extreme scenarios. We analyze the impact of possible distributional choices for the SHP. In a multivariate setting, we hint at the possible introduction of dependent SHP processes, which potentially lead to nonlinear dependence among the P\&L processes and therefore to tail dependence across assets in the portfolio, although this may require drastic choices on the SHP distributions. We also find that increasing dependence as measured by Kendall's tau through common SHPs appears to be unfeasible. We finally discuss potential developments following future availability of market data. This chapter is a refined version of the original working paper by Brigo and Nordio (2010) [14].
\end{abstract}

\section{Introduction}

According to the Interaction between Market and Credit Risk (IMCR) research group of the Basel Committee on Banking Supervision (BCBS) [5], liquidity conditions interact with market risk and credit risk through the horizon over which assets can be liquidated. To face the impact of market liquidity risk, risk managers agree in adopting a longer holding period to calculate the market VaR, for instance 10 business

\author{
D. Brigo $(\varangle)$ \\ Department of Mathematics, Imperial College London, 180 Queen's Gate, \\ London SW7 2AZ, UK \\ e-mail: damiano.brigo@imperial.ac.uk \\ C. Nordio \\ Risk Management, Banco Popolare, Milan, Italy \\ e-mail: claudio.nordio@bancopopolare.it


days instead of 1 ; recently, BCBS has prudentially stretched such liquidity horizon to 3 months [6]. However, even the IMCR group pointed out that the liquidity of traded products can vary substantially over time and in unpredictable ways, and moreover, IMCR studies suggest that banks' exposures to market risk and credit risk vary with liquidity conditions in the market. The former statement suggests a stochastic description of the time horizon over which a portfolio can be liquidated, and the latter highlights a dependence issue.

We can start by saying that probably the holding period of a risky portfolio is neither 10 business days nor 3 months; it could, for instance, be 10 business days with probability $99 \%$ and 3 months with probability $1 \%$. This is a very simple assumption but it may have already interesting consequences. Indeed, given the FSA (now Bank of England) requirement to justify liquidity horizon assumptions for the Incremental Risk Charge modeling, a simple example with the two-points liquidity horizon distribution that we develop below could be interpreted as a mixture of the distribution under normal conditions and of the distribution under stressed and rare conditions. In the following we will assume no transaction costs, in order to fully represent the liquidity risk through the holding period variability. Indeed, if we introduce a process describing the dynamics of such liquidity conditions, for instance,

- the process of time horizons over which the risky portfolio can be fully bought or liquidated,

then the P\&L is better defined by the returns calculated over such stochastic time horizons instead of a fixed horizon (say daily, weekly or monthly basis). We will use the "stochastic holding period" (SHP) acronym for that process, which belongs to the class of positive processes largely used in mathematical finance. We define the liquidity-adjusted VaR or Expexted Shortfall (ES) of a risky portfolio as the VaR or ES of portfolio returns calculated over the horizon defined by the SHP process, which is the 'operational time' along which the portfolio manager must operate, in contrast to the 'calendar time' over which the risk manager usually measures VaR.

\subsection{Earlier Literature}

Earlier literature on extending risk measures to liquidity includes several studies. Jarrow and Subramanian [17], Bangia et al. [4], Angelidis and Benos [3], Jarrow and Protter [18], Stange and Kaserer [25], Ernst, Stange and Kaserer [15], among others, propose different methods of extending risk measures to account for liquidity risk. Bangia et al. [4] classify market liquidity risk into two categories: (a) the exogenous illiquidity that depends on general market conditions is common to all market players and is unaffected by the actions of any one participant and (b) the endogenous illiquidity, which is specific to one's position in the market, varies across different market players and is mainly related to the impact of the trade size on the bid-ask spread. Bangia et al. [4] and Ernst et al. [15] only consider the exogenous illiquidity 
risk and propose a liquidity adjusted VaR measure built using the distribution of the bid-ask spreads. The other mentioned studies model and account for endogenous risk in the calculation of liquidity adjusted risk measures. In the context of the coherent risk measures literature, the general axioms a liquidity measure should satisfy are discussed in [1]. In that work coherent risk measures are defined on the vector space of portfolios (rather than on portfolio values). A key observation is that the portfolio value can be a nonlinear map on the space of portfolios, motivating the introduction of a nonlinear value function depending on a notion of liquidity policy based on a general description of the microstructure of illiquid markets.

As mentioned earlier, bid-ask spreads have been used to assess liquidity risk. While bid-ask spreads are certainly an important measure of liquidity, they are not the only one. In the Credit Default Swap (CDS) space, for example, Predescu et al. [22] have built a statistical model that associates an ordinal liquidity score with each CDS reference entity. The liquidity score is built using well-known liquidity indicators such as the already mentioned bid-ask spreads but also using other less accessible predictors of market liquidity such as number of active dealers quoting a reference entity, staleness of quotes of individual dealers, and dispersion in midquotes across market dealers. The bid-ask spread is used essentially as an indicator of market breadth; the presence of orders on both sides of the trading book corresponds to tighter bid-ask spreads. Dispersion of mid-quotes across dealers is a measure of price uncertainty about the actual CDS price. Less liquid names are generally associated with more price uncertainty and thus large dispersion. The third liquidity measure that is used in Predescu et al. [22] aggregates the number of active dealers and the individual dealers' quote staleness into an (in)activity measure, which is meant to be a proxy for CDS market depth. Illiquidity increases if any of the liquidity predictors increases, keeping everything else constant. Therefore, liquid (less liquid) names are associated with smaller (larger) liquidity scores. CDS liquidity scores are now offered commercially by Fitch Solutions and as of 2009 provided a comparison of relative liquidity of over 2,400 reference entities in the CDS market globally, mainly concentrated in North America, Europe, and Asia. The model estimation and the model generated liquidity scores are based upon the Fitch CDS Pricing Service database, which includes single-name CDS quotes on over 3,000 entities, corporates, and sovereigns across about two dozen broker-dealers back to 2000. This approach and the related results, further highlighting the connection between liquidity and credit quality/rating, are summarized in [14], who further review previous research on liquidity components in the pricing space for CDS.

Given the above indicators of liquidity risk, the SHP process seems to be naturally associated with the staleness/inactivity measure. However, one may argue that the random holding period also embeds market impact and bid-ask spreads. Indeed, traders will consider closing a position or a portfolio also in terms of cost. If bidask spreads cause the immediate closure of a position to be too expensive, market operators might wait for bid-asks to move. This will impact the holding period for the relevant position. If we take for granted that the risk manager will not try to model the detailed behavior of traders, then the stochastic holding period becomes a reduced form process for the risk manager, which will possibly incapsulate a number 
of aspects on liquidity risk. Ideally, as our understanding of liquidity risk progresses, we can move to a more structural model where the dynamics of the SHP is explained in terms of market prices and liquidity proxies, including market impact, bid-ask spreads, and asset prices. However, in this work we sketch the features the resulting model could have in a reduced form spirit.

This prompts us to highlight a further feature that we should include in future developments of the model introduced here: we should explicitly include dependence between price levels and holding periods, since liquidity is certainly related to the level of prices in the market.

\subsection{Different Risk Horizons Are Acknowledged by BCBS}

The Basel Committee came out with a recommendation on multiple holding periods for different risk factors in 2012 in [7]. This document states that

The Committee is proposing that varying liquidity horizons be incorporated in the market risk metric under the assumption that banks are able to shed their risk at the end of the liquidity horizon.[...]. This proposed liquidation approach recognises the dynamic nature of banks trading portfolios but, at the same time, it also recognises that not all risks can be unwound over a short time period, which was a major flaw of the 1996 framework.

Further on, in Annex 4, the document details a sketch of a possible solution: assign a different liquidity horizon to risk factors of different types. While this is a step forward, it can be insufficient. How is one to decide the horizon for each risk factor, and especially how is one to combine the different estimates for different horizons for assets in the same portfolio into a consistent and logically sound way? Our random holding period approach allows one to answer the second question, but more generally none of the above works focuses specifically on our setup with random holding period, which represents a simple but powerful idea to include liquidity in traditional risk measures such as Value at Risk or Expected Shortfall. Our idea was first proposed in 2010 in [13].

When analyzing multiple positions, holding periods can be taken to be strongly dependent, in line with the first classification (a) of Bangia et al. [4] above, or independent, so as to fit the second category (b). We will discuss whether adding dependent holding periods to different positions can actually add dependence to the position returns.

The paper is organized as follows. In order to illustrate the SHP model, first in a univariate case (Sect.2) and then in a bivariate one (Sect.3), it is considerably easier to focus on examples on (log)normal processes. A brief colloquial hint at positive processes is presented in Sect.2, to deepen the intuition of the impact on risk measures of introducing a SHP process. Across Sects. 3 and 4, where we try to address the issue of calibration, we outline a possible multivariate model which could be adopted, in line of principle, in a top-down approach to risk integration in order to include the liquidity risk and its dependence on other risks. 
Table 1 Simplified discrete SHP

\begin{tabular}{l|l}
\hline Holding period & Probability \\
\hline 10 & 0.99 \\
\hline 75 & 0.01 \\
\hline
\end{tabular}

Finally, we point out that this paper is meant as a proposal to open a research effort in stochastic holding period models for risk measures. This paper contains several suggestions on future developments, depending on an increased availability of market data. The core ideas on the SHP framework, however, are presented in this opening paper.

\section{The Univariate Case}

Let us suppose that we have to calculate the VaR of a market portfolio whose value at time $t$ is $V_{t}$. We call $X_{t}=\ln V_{t}$, so that the $\log$ return on the portfolio value at time $t$ over a period $h$ is

$$
X_{t+h}-X_{t}=\ln \left(V_{t+h} / V_{t}\right) \approx \frac{V_{t+h}-V_{t}}{V_{t}} .
$$

In order to include liquidity risk, the risk manager decides that a realistic, simplified statistics of the holding period in the future will be the one given in Table 1. To estimate liquidity-adjusted $\mathrm{VaR}$ say at time 0 , the risk manager will perform a number of simulations of $V_{0+H_{0}}-V_{0}$ with $H_{0}$ randomly chosen by the statistics above, and finally will calculate the desired risk measure from the resulting distribution. If the log-return $X_{T}-X_{0}$ is normally distributed with zero mean and variance $T$ for deterministic $T$ (e.g., a Brownian motion, i.e., a Random walk), then the risk manager could simplify the simulation using $X_{0+H_{0}}-\left.X_{0}\right|_{H_{0}} \stackrel{d}{\sim} \sqrt{H_{0}}\left(X_{1}-X_{0}\right)$ where $\left.\right|_{H_{0}}$ denotes "conditional on $H_{0}$ ". With this practical exercise in mind, let us generalize this example to a generic $t$.

\subsection{A Brief Review on the Stochastic Holding Period Framework}

A process for the risk horizon at time $t$, i.e., $t \mapsto H_{t}$, is a positive stochastic process modeling the risk horizon over time. We have that the risk measure at time $t$ will be taken on the change in value of the portfolio over this random horizon. If $X_{t}$ is the $\log$-value of the portfolio at time $t$, we have that the risk measure at time $t$ is to be taken on the log-return

$$
X_{t+H_{t}}-X_{t}
$$


For example, if one uses a $99 \%$ Value at Risk (VaR) measure, this will be the 1st percentile of $X_{t+H_{t}}-X_{t}$. The request that $H_{t}$ be just positive means that the horizon at future times can both increase and decrease, meaning that liquidity can vary in both directions.

There are a large number of choices for positive processes: one can take lognormal processes with or without mean reversion, mean reverting square root processes, squared Gaussian processes, all with or without jumps. This allows one to model the holding period dynamics as mean reverting or not, continuous or with jumps, and with thinner or fatter tails. Other examples are possible, such as Variance Gamma or mixture processes, or Levy processes. See for example [11, 12].

\subsection{Semi-analytic Solutions and Simulations}

Going back to the previous example, let us suppose that

Assumption 1 The increments $X_{t+1 y}-X_{t}$ are logarithmic returns of an equity index, normally distributed with annual mean and standard deviation, respectively, $\mu_{1 y}=-1.5 \%$ and $\sigma_{1 y}=30 \%$.

We suppose an exposure of 100 in domestic currency.

Before running the simulation, we recall some basic notation and formulas.

The portfolio log-returns under random holding period at time 0 can be written as

$$
\mathbb{P}\left[X_{H_{0}}-X_{0}<x\right]=\int_{0}^{\infty} \mathbb{P}\left[X_{h}-X_{0}<x\right] \mathrm{d} F_{H, 0}(h)
$$

i.e., as a mixture of Gaussian returns, weighted by the holding period distribution. Here $F_{H, t}$ denotes the cumulative distribution function of the holding period at time $t$, i.e., of $H_{t}$.

Remark 1 (Mixtures for heavy-tailed and skewed distributions). Mixtures of distributions have been used for a long time in statistics and may lead to heavy tails, allowing for modeling of skewed distributions and of extreme events. Given the fact that mixtures lead, in the distributions space, to linear (convex) combinations of possibly simple and well-understood distributions, they are tractable and easy to interpret. The literature on mixtures is enormous and it is impossible to do justice to all this literature here. We just hint at the fact that static mixtures of distributions had been postulated in the past to fit option prices for a given maturity, see for example [24], where a mixture of normal densities for the density of the asset log-returns under the pricing measure is assumed, and subsequently [8, 16, 20]. In the last decade $[2,9,10]$ have extended the mixture distributions to fully dynamic arbitrage-free stochastic processes for asset prices. 
Table 2 SHP distributions and market risk

\begin{tabular}{l|l|l|l|l}
\hline Holding period & VaR 99.96\% & (Analytic) & ES 99.96\% & (Analytic) \\
\hline Constant 10 b.d. & 20.1 & $(20.18)$ & 21.7 & $(21.74)$ \\
\hline Constant 75 b.d. & 55.7 & $(55.54)$ & 60.0 & $(59.81)$ \\
\hline SHP (Bernoulli 10/75, $\left.p_{10}=0.99\right)$ & 29.6 & $(29.23)$ & 36.1 & $(35.47)$ \\
\hline
\end{tabular}

Going back to our notation, $\mathrm{VaR}_{t, h, c}$ and $\mathrm{ES}_{t, h, c}$ are the value at risk and expected shortfall, respectively, for a horizon $h$ at confidence level $c$ at time $t$, namely

$\mathbb{P}\left\{X_{t+h}-X_{t}>-\mathrm{VaR}_{t, h, c}\right\}=c, \mathrm{ES}_{t, h, c}=-\mathbb{E}\left[X_{t+h}-X_{t} \mid X_{t+h}-X_{t} \leq-\mathrm{VaR}_{t, h, c}\right]$.

We now recall the standard result on VaR and ES under Gaussian returns in deterministic calendar time.

Proposition 1 (VaR and ES with Gaussian log-returns on a deterministic risk horizon $h$ ) In the Gaussian log-returns case where

$$
X_{t+h}-X_{t} \text { is normally distributed with mean } \mu_{t, h} \text { and standard deviation } \sigma_{t, h}
$$

we obtain

$$
\operatorname{VaR}_{t, h, c}=-\mu_{t, h}+\Phi^{-1}(c) \sigma_{t, h}, E S_{t, h, c}=-\mu_{t, h}+\sigma_{t, h} p\left(\Phi^{-1}(c)\right) /(1-c)
$$

where $p$ is the standard normal probability density function and $\Phi$ is the standard normal cumulative distribution function.

In the following we will calculate VaR and Expected Shortfall referred to a confidence level of $99.96 \%$, calculated over the fixed time horizons of 10 and 75 business days, and under SHP process with statistics given by Table 1, using Monte Carlo simulations. Each year has 250 (working) days. The results are presented in Table 2.

More generally, we may derive the VaR and ES formulas for the case where $H_{t}$ is distributed according to a general distribution

$$
\mathbb{P}\left(H_{t} \leq x\right)=F_{H, t}(x), \quad x \geq 0
$$

and

$$
\mathbb{P}\left(X_{t+h}-X_{t} \leq x\right)=F_{X, t, h}(x)
$$

Definition 1 (VaR and ES under Stochastic Holding Period) We define VaR and ES under a random horizon $H_{t}$ at time $t$ and for a confidence level $c$ as

$$
\mathbb{P}\left\{X_{t+H_{t}}-X_{t}>-\mathrm{VaR}_{H, t, c}\right\}=c, \mathrm{ES}_{H, t, c}=-\mathbb{E}\left[X_{t+H_{t}}-X_{t} \mid X_{t+H_{t}}-X_{t} \leq-\mathrm{VaR}_{H, t, c}\right]
$$


We point out that the order of time/confidence/horizon arguments in the VaR and ES definitions is different in the Stochastic Holding Period case. This is to stress the different setting with respect to the fixed holding period case.

We have immediately the following:

Proposition 2 (VaR and ES for SHP independent of returns in deterministic calendar time) Assume that $H$ is independent of the log returns of $X$ in deterministic calendar time. Using the tower property of conditional expectation it is immediate to prove that such a case $V_{a R_{H, c}}$ obeys the following equation:

$$
\int_{0}^{\infty}\left(1-F_{X, t, h}\left(-\operatorname{VaR}_{H, t, c}\right)\right) \mathrm{d} F_{H, t}(h)=c
$$

whereas $E S_{H, t, c}$ is given by

$$
E S_{H, t, c}=-\frac{1}{1-c} \int_{0}^{\infty} \mathbb{E}\left[X_{t+h}-X_{t} \mid X_{t+h}-X_{t} \leq-\operatorname{VaR}_{H, t, c}\right] \operatorname{Prob}\left(X_{t+h}-X_{t} \leq-\operatorname{VaR}_{H, t, c}\right) \mathrm{d} F_{H, t}(h)
$$

For the specific Gaussian case (1) we have

$$
\begin{gathered}
\int_{0}^{\infty} \Phi\left(\frac{\mu_{t, h}+V a R_{H, t, c}}{\sigma_{t, h}}\right) \mathrm{d} F_{H, t}(h)=c \\
E S_{H, t, c}=\frac{1}{1-c} \int_{0}^{\infty}\left[-\mu_{t, h} \Phi\left(\frac{-\mu_{t, h}-V a R_{H, t, c}}{\sigma_{t, h}}\right)+\sigma_{t, h} p\left(\frac{-\mu_{t, h}-V a R_{H, t, c}}{\sigma_{t, h}}\right)\right] \mathrm{d} F_{H, t}(h)
\end{gathered}
$$

Notice that in general one can try and obtain the quantile $\mathrm{VaR}_{H, t, c}$ for the random horizon case by using a root search, and subsequently compute also the expected shortfall. Careful numerical integration is needed to apply these formulas for general distributions of $H_{t}$. The case of Table 2 is somewhat trivial, since in the case where $H_{0}$ is as in Table 1 integrals reduce to summations of two terms.

We note also that the maximum difference, both in relative and absolute terms, between ES and VaR is reached by the model under random holding period $H_{0}$. Under this model the change in portfolio value shows heavier tails than under a single deterministic holding period. In order to explore the impact of SHP's distribution tails on the liquidity-adjusted risk, in the following we will simulate SHP models with $H_{0}$ distributed as an Exponential, an Inverse Gamma distribution ${ }^{1}$ and a Generalized

\footnotetext{
${ }^{1}$ Obtained by rescaling a distribution $\operatorname{IG}\left(\frac{v}{2}, \frac{v}{2}\right)$ with $v=3$. Before rescaling, setting $\alpha=v / 2$, the inverse gamma density is $f(x)=(1 / \Gamma(\alpha))(\alpha)^{\alpha} x^{-\alpha-1} \mathrm{e}^{-\alpha / x}, x>0, \alpha>0$, with expected value $\alpha /(\alpha-1)$. We rescale this distribution by $k=8.66 /(\alpha /(\alpha-1))$ and take for $H_{0}$ the random variable with density $f(x / k) / k$.
} 
Pareto distribution ${ }^{2}$ having parameters calibrated in order to obtain a sample with the same $99 \%$-quantile of 75 business days. The results are in Table 3.

The SHP process changes the statistical nature of the P\&L process: the heavier the tails of the SHP distribution, the heavier the tails of P\&L distribution. Notice that our Pareto distribution has tails going to 0 at infinity with exponent around 3 , as one can see immediately by differentiation of the cumulative distribution function, whereas our inverse gamma has tails going to 0 at infinity with exponent about 2.5. In this example we have that the tails of the inverse gamma are heavier, and indeed for that distribution VaR and ES are larger and differ from each other more. This can change of course if we take different parameters in the two distributions.

\section{Dependence Modeling: A Bivariate Case}

Within multivariate modeling, using a common SHP for many normally distributed risks leads to dynamical versions of the so-called normal mixtures and normal meanvariance mixtures [19].

Assumption 2 In this section we assume that different assets have the same random holding period, thus testing an extreme liquidity dependence scenario. We will briefly discuss relaxing this assumption at the end of this section. We further assume that the stochastic holding period process is independent of the log returns of assets in deterministic calendar time.

Let the $\log$ returns (recall $X_{t}^{i}=\ln V_{t}^{i}$, with $V_{t}^{i}$ the value at time $t$ of the ith asset)

$$
X_{t+h}^{1}-X_{t}^{1}, \ldots, X_{t+h}^{m}-X_{t}^{m}
$$

be normals with means $\mu_{t, h}^{1}, \ldots, \mu_{t, h}^{m}$ and covariance matrix $Q_{t, h}$.

Then

$$
\begin{aligned}
\mathbb{P} & {\left[X_{t+H_{t}}^{1}-X_{t}^{1}<x_{1}, \ldots, X_{t+H_{t}}^{m}-X_{t}^{m}<x_{m}\right] } \\
& =\int_{0}^{\infty} \mathbb{P}\left[X_{t+h}^{1}-X_{t}^{1}<x_{1}, \ldots, X_{t+h}^{m}-X_{t}^{m}<x_{m}\right] \mathrm{d} F_{H, t}(h)
\end{aligned}
$$

is distributed as a mixture of multivariate normals, and a portfolio $V_{t}$ of the assets $1,2, \ldots, m$ whose log-returns $X_{t+h}-X_{t}\left(X_{t}=\ln V_{t}\right)$ are a linear weighted combination $w_{1}, \ldots, w_{m}$ of the single asset $\log$-returns $X_{t+h}^{i}-X_{t}^{i}$ would be distributed as

\footnotetext{
${ }^{2}$ With scale parameter $k=9$ and shape parameter $\alpha=2.0651$, with cumulative distribution function $F(x)=1-\left(\frac{k}{k+x}\right)^{\alpha}, x \geq 0$, this distribution has moments up to order $\alpha$. So the smaller $\alpha$, the fatter the tails. The mean is, if $\alpha>1, \mathbb{E}\left[H_{0}\right]=k /(\alpha-1)$.
} 


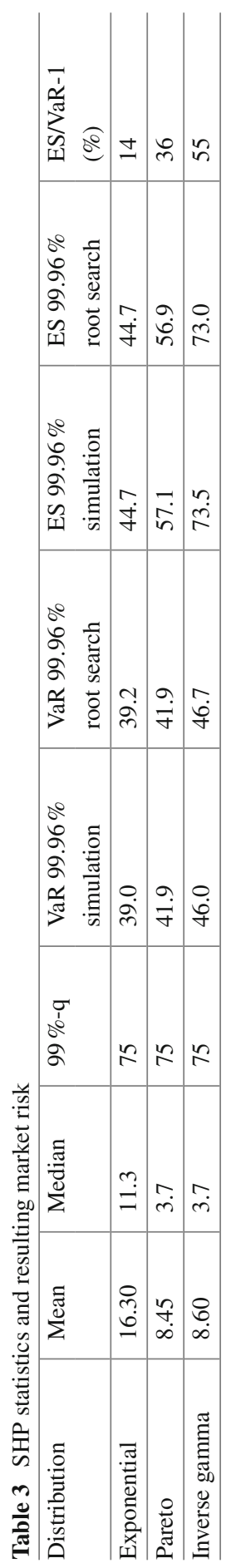


$\mathbb{P}\left[X_{t+H_{t}}-X_{t}<z\right]=\int_{0}^{\infty} \mathbb{P}\left[w_{1}\left(X_{t+h}^{1}-X_{t}^{1}\right)+\cdots+w_{m}\left(X_{t+h}^{m}-X_{t}^{m}\right)<z\right] \mathrm{d} F_{H, t}(h)$

In particular, in analogy with the unidimensional case, the mixture may potentially generate skewed and fat-tailed distributions, but when working with more than one asset this has the further implication that VaR is not guaranteed to be subadditive on the portfolio. Then the risk manager who wants to take into account SHP in such a setting should adopt a coherent measure like Expected Shortfall.

A natural question at this stage is whether the adoption of a common SHP can add dependence to returns that are jointly Gaussian under deterministic calendar time, perhaps to the point of making extreme scenarios on the joint values of the random variables possible.

Before answering this question, one needs to distinguish extreme behavior in the single variables and in their joint action in a multivariate setting. Extreme behavior on the single variables is modeled, for example, by heavy tails in the marginal distributions of the single variables. Extreme behavior in the dependence structure of, say, two random variables is achieved when the two random variables tend to take extreme values in the same direction together. This is called tail dependence, and one can have both upper tail dependence and lower tail dependence. More precisely, but still loosely speaking, tail dependence expresses the limiting proportion according to which the first variable exceeds a certain level given that the second variable has already exceeded that level. Tail dependence is technically defined through a limit, so that it is an asymptotic notion of dependence. For a formal definition we refer, for example, to [19]. "Finite" dependence, as opposed to tail, between two random variables is best expressed by rank correlation measures such as Kendall's tau or Spearman's rho.

We discuss tail dependence first. In case the returns of the portfolio assets are jointly Gaussian with correlations smaller than one, the adoption of a common random holding period for all assets does not add tail dependence, unless the commonly adopted random holding period has a distribution with power tails. Hence, if we want to rely on one of the random holding period distributions in our examples above to introduce upper and lower tail dependence in a multivariate distribution for the assets returns, we need to adopt a common random holding period for all assets that is Pareto or Inverse Gamma distributed. Exponentials, Lognormals, or discrete Bernoulli distributions would not work. This can be seen to follow from properties of the normal variance-mixture model, see for example [19], p. 212 and also Sect. 7.3.3.

A more specific theorem that fits our setup is Theorem 5.3.8 in [23]. We can write it as follows with our notation.

Proposition 3 (A common random holding period with less than power tails does not add tail dependence to jointly Gaussian returns) Assume the log returns to be $W_{t}^{i}=\ln V_{t}^{i}$, with $V_{t}^{i}$ the value at time $t$ of the ith asset, $i=1,2$, where

$$
W_{t+h}^{1}-W_{t}^{1}, W_{t+h}^{2}-W_{t}^{2}
$$


are two correlated Brownian motions, i.e., normals with zero means, variances $h$, and instantaneous correlation less than 1 in absolute value:

$$
\mathrm{d}\left\langle W^{1}, W^{2}\right\rangle_{t}=\mathrm{d} W_{t}^{1} \mathrm{~d} W_{t}^{2}=\rho_{1,2} \mathrm{~d} t, \quad\left|\rho_{1,2}\right|<1 .
$$

Then adding a common nonnegative random holding period $H_{0}$ independent of $W$ 's leads to tail dependence in the returns

$$
W_{H_{0}}^{1}, W_{H_{0}}^{2}
$$

if and only if $\sqrt{H_{0}}$ is regularly varying at $\infty$ with index $\alpha>0$.

Theorem 5.3.8 in [23] also reports an expression for the tail dependence coefficients as functions of $\alpha$ and of the survival function of the student $t$ distribution with $\alpha+1$ degrees of freedom.

Summarizing, if we work with power tails, the heavier the tails of the common holding period process $H$, the more one may expect tail dependence to emerge for the multivariate distribution: by adopting a common SHP for all risks, dependence could potentially appear in the whole dynamics, in agreement with the fact that liquidity risk is a systemic risk.

We now turn to finite dependence, as opposed to tail dependence. First, we note the well-known elementary but important fact that one can have two random variables with very high dependence but without tail dependence. Or one can have two random variables with tail dependence but small finite dependence. For example, if we take two jointly Gaussian Random variables with correlation 0.999999, they are clearly quite dependent on each other but they will not have tail dependence, even if a rank correlation measure such as Kendall's $\tau$ would be 0.999 , still very close to 1 , characteristic of the co-monotonic case. This is a case with zero tail dependence but very high finite dependence. On the other hand, take a bivariate student $t$ distribution with few degrees of freedom and correlation parameter $\rho=0.1$. In this case the two random variables have positive tail dependence and it is known that Kendall's tau for the two random variables is

$$
\tau=\frac{2}{\pi} \arcsin (\rho) \approx 0.1
$$

which is the same tau one would get for two standard jointly Gaussian random variables with correlation $\rho$. This tau is quite low, showing that one can have positive tail dependence while having very small finite dependence.

The above examples point out that one has to be careful in distinguishing large finite dependence and tail dependence.

A further point of interest in the above examples comes from the fact that the multivariate student $t$ distribution can be obtained by the multivariate Gaussian distribution when adopting a random holding period given by an inverse gamma distribution (power tails). We deduce the important fact that in this case a common 
random holding period with power tails adds positive tail dependence but not finite dependence.

In fact, one can prove a more general result easily by resorting to the tower property of conditional expectation and from the definition of tau based on independent copies of the bivariate random vector whose dependence is being measured. One has the following "no go" theorem for increasing Kendall's tau of jointly Gaussian returns through common random holding periods, regardless of the tail's power.

Proposition 4 (A common random holding period does not alter Kendall's tau for jointly Gaussian returns) Assumptions as in Proposition 3 above. Then adding a common nonnegative random holding period $H_{0}$ independent of $W$ 's leads to the same Kendall's tau for

$$
W_{H_{0}}^{1}, W_{H_{0}}^{2}
$$

as for the two returns

$$
W_{t}^{1}, W_{t}^{2}
$$

for a given deterministic time horizon $t$.

Summing up, this result points out that adding further finite dependence through common SHPs, at least as measured by Kendall's tau, can be impossible if we start from Gaussian returns. A different popular rank correlation measure, Spearman's rho, does not coincide for the bivariate $t$ and Gaussian cases though, so that it is not excluded that dependence could be added in principle though dependent holding periods, at least if we measured dependence with Spearman's $\rho$. This is under investigation.

More generally, at least from a theoretical point of view, it could be interesting to model other kinds of dependence than the one stemming purely from a common holding period (with power tails). In the bivariate case, for example, one could have two different holding periods that are themselves dependent on each other in a less simplistic way, for example through a common factor structure, rather than being just identical. In this case it would be interesting to study the tail dependence implications and also finite dependence as measured by Spearman's rho.

We will investigate this aspect in further research, but increasing dependence may require, besides the adoption of power tail laws for the random holding periods, abandoning the Gaussian distribution for the basic assets under deterministic calendar time.

A further aspect worth investigating is the possibility to calculate semi-closed form risk contributions to VaR and ES under SHP along the lines suggested in [26], and to investigate the Euler principle as in [27, 28].

\section{Calibration with Liquidity Data}

We are aware that multivariate SHP modeling is a purely theoretical exercise and that we just hinted at possible initial developments above. Nonetheless, a lot of financial data is being collected by regulators, providers, and rating agencies, together with 
a consistent effort on theoretical and statistical studies. This will possibly result in available synthetic indices of liquidity risk grouped by region, market, instrument type, etc. For instance, Fitch already calculates market liquidity indices on CDS markets worldwide, on the basis of a scoring proprietary model [14].

\subsection{Dependencies Between Liquidity, Credit, and Market Risk}

It could be an interesting exercise to calibrate the dependence structure (e.g., copula function) between a liquidity index (like the Fitch's one), a credit index (like iTRAXX), and a market index (for instance Eurostoxx50) in order to measure the possible (nonlinear) dependence between the three. The risk manager of a bank could use the resulting dependence structure within the context of risk integration, in order to simulate a joint dynamics as a first step, to estimate later on the whole liquidity-adjusted VaR/ES by assuming co-monotonicity between the variations of the liquidity index and of the SHP processes.

\subsection{Marginal Distributions of SHPs}

A lot of information on SHP 'extreme' statistics of an OTC derivatives portfolio could be collected from the statistics, across Lehman's counterparties, of the time lags between the Lehman's Default Event Date and the trade dates of any replacement transaction. The data could give information on the marginal distribution of the SHP of a portfolio, in a stressed scenario, by assuming a statistical equivalence between data collected 'through the space' (across Lehman's counterparties) and 'through the time' under i.i.d. hypothesis. ${ }^{3}$ The risk manager of a bank could examine a more specific and non-distressed dataset by collecting information on the ordinary operations of the business units.

\section{Conclusions}

Within the context of risk integration, in order to include liquidity risk in the whole portfolio risk measures, a stochastic holding period (SHP) model can be useful, being versatile, easy to simulate, and easy to understand in its inputs and outputs. In a single-portfolio framework, as a consequence of introducing an SHP model, the statistical distribution of $\mathrm{P} \& \mathrm{~L}$ moves to possibly heavier tailed and skewed mixture distributions. In a multivariate setting, the dependence among the SHP processes to which marginal P\&L are subordinated, may lead to dependence on the latter under drastic choices of the SHP distribution, and in general to heavier tails on the total

\footnotetext{
${ }^{3}$ A similar approach is adopted in [21] within the context of operational risk modeling.
} 
P\&L distribution. At present, lack of synthetic and consensually representative data forces to a qualitative top-down approach, but it is straightforward to assume that this limit will be overcome in the near future.

Acknowledgments This paper reflects the authors' opinions and not necessarily those of their current and past employers. The authors are grateful to Dirk Tasche for helpful suggestions and correspondence on a first version, to Giacomo Scandolo for further comments and correspondence, and to an anonymous referee and the Editors for important suggestions that helped in improving the paper.

Open Access This chapter is distributed under the terms of the Creative Commons Attribution Noncommercial License, which permits any noncommercial use, distribution, and reproduction in any medium, provided the original author(s) and source are credited.

\section{References}

1. Acerbi, C., Scandolo, G.: Liquidity risk theory and coherent measures of risk. Quant. Financ. 8(7), 681-692 (2008)

2. Alexander, C.: Normal mixture diffusion with uncertain volatility: modelling short and longterm smile effects. J. Bank. Financ. 28(12), 2957-2980 (2004)

3. Angelidis, T., Benos, A.: Liquidity adjusted value-at-risk based on the components of the bid-ask spread. Working paper, available at http://ssrn.com/abstract=661281 (2005)

4. Bangia, A., Diebold, F.X., Schuermann, T., Stroughair, J.D.: Modeling liquidity risk with implications for traditional market risk measurement and management. Working paper, Financial Institutions Center at The Wharton School (1999)

5. Basel Committee on Banking Supervision. Findings on the interaction of market and credit risk, BCBS working paper No 16 May 2009, available at http://www.bis.org (2009)

6. Basel Committee on Banking Supervision. Guidelines for computing capital for incremental risk in the trading book, BCBS guidelines, July 2009, available at http://www.bis.org (2009)

7. Basel Committee on Banking Supervision. Consultative document: fundamental review of the trading book, BIS, May 2012, available at http://www.bis.org/publ/bcbs219.pdf (2012)

8. Bhupinder, B.: Implied risk-neutral probability density functions from option prices: a central bank perspective. In: Knight, J., Satchell, S. (eds.) Forecasting Volatility in the Financial Markets, pp. 137-167. Butterworth Heinemann, Oxford (1998)

9. Brigo, D., Mercurio, F.: Displaced and mixture diffusions for analytically-tractable smile models. In: Geman, H., Madan, D.B., Pliska, S.R., Vorst, A.C.F. (eds.) Mathematical FinanceBachelier Congress 2000. Springer, Berlin (2001)

10. Brigo, D., Mercurio, F., Rapisarda, F.: Smile at uncertainty, risk, May issue (2004)

11. Brigo, D., Dalessandro, A., Neugebauer, M., Triki, F.: A stochastic processes toolkit for risk management: geometric Brownian motion, jumps, GARCH and variance gamma models. J. Risk Manag. Financ. Inst. 2(4), 365-393 (2009)

12. Brigo, D., Dalessandro, A., Neugebauer, M., Triki, F.: A stochastic processes toolkit for risk management. Mean reversing processes and jumps. J. Risk Manag. Financ. Inst. 3, 1 (2009)

13. Brigo, D., Nordio, C.: Liquidity-adjusted market risk measures with stochastic holding period. Available at http://arxiv.org/abs/1009.3760 and http://ssrn.com/abstract=1679698 (2010)

14. Brigo, D., Predescu, M., Capponi, A.: Liquidity modeling for credit default swaps: an overview. In: Bielecki, Brigo, Patras (eds.) Credit Risk Frontiers: Sub prime crisis, Pricing and Hedging, CVA, MBS, Ratings and Liquidity, pp. 587-617. Wiley/Bloomberg Press, See also http://ssrn. com/abstract=1564327 (2010) 
15. Ernst, C., Stange, S., Kaserer, C.: Accounting for non-normality in liquidity risk. Available at http://ssrn.com/abstract=1316769 (2009)

16. Guo, C.: Option pricing with heterogeneous expectations. Financ. Rev. 33, 81-92 (1998)

17. Jarrow, R., Subramanian, A.: Mopping up liquidity. RISK 10(10), 170-173 (1997)

18. Jarrow, R., Protter, P.: Liquidity Risk and Risk Measure Computation. Working paper, Cornell University (2005)

19. McNeil, A.J., Frey, R., Embrechts, P.: Quantitative Risk Management. Princeton University Press, New Jersey (2005)

20. Melick, W.R., Thomas, C.P.: Recovering an asset's implied PDF from option prices: an application to crude oil during the Gulf crisis. J. Financ. Quant. Anal. 32, 91-115 (1997)

21. Moscadelli, M.: The modelling od operational risk: experience with the analysis of the data collected by the Base Committee, Banca d'Italia, Temi di discussione del Servizio Studi, Number 517 (July 2004), available at http://www.bancaditalia.it/pubblicazioni/econo/temidi (2004)

22. Predescu, M., Thanawalla, R., Gupton, G., Liu, W., Kocagil, A., Reyngold, A.: Measuring CDS liquidity. Fitch Solutions Presentation at the Bowles Symposium, Georgia State University, 12(2009)

23. Prestele, C.: Credit portfolio modelling with elliptically contoured distributions. Approximation, Pricing, Dynamisation. Doctoral dissertation, Ulm University. Available at http://vts.uniulm.de/docs/2007/6093/vts_6093_8209.pdf (2007)

24. Ritchey, R.J.: Call option valuation for discrete normal mixtures. J. Financ. Res. 13, 285-296 (1990)

25. Stange, S., Kaserer, C.: Why and how to integrate liquidity risk into a VaR-framework. CEFS working paper 2008 No. 10, available at http://ssrn.com/abstract=1292289 (2008)

26. Tasche, D.: Measuring sectoral diversification in an asymptotic multi-factor framework. J. Credit Risk 2(3), 33-55 (2006)

27. Tasche, D.: Capital allocation to business units and sub-portfolios: the euler principle. In: Resti, A., (ed.) Pillar II in the New Basel Accord: The Challenge of Economic Capital, Risk Books, pp. 423-453 (2008)

28. Tasche, D.: Capital allocation for credit portfolios with kernel estimators. Quant. Financ. 9(5), 581-595 (2009) 